\title{
Compreensão de Propriedades da Mediana e da Amplitude por Futuros Professores dos Primeiros Anos
}

\author{
José António Fernandes \\ jfernandes@ie.uminho.pt \\ https://orcid.org/0000-0003-2015-160X \\ Universidade do Minho (UM) \\ Braga, Portugal
}

Recibido: 14/09/2020 Aceptado: 09/02/2021

\begin{abstract}
Resumo
Neste artigo estuda-se a compreensão de estudantes, futuros professores dos primeiros anos escolares, de propriedades das estatísticas mediana e amplitude total. No estudo participaram 34 estudantes que frequentavam o 2. ${ }^{\circ}$ ano do curso de Licenciatura em Educação Básica de uma universidade do norte de Portugal. Os estudantes resolveram várias questões, em contexto de sala de aula e para efeitos de avaliação formal, envolvendo situações-problema de Estatística Descritiva, das quais iremos apresentar aqui apenas duas. Nessas questões, com dois itens cada, avaliam-se possíveis alterações ocorridas na mediana e na amplitude total quando se adiciona um valor constante a todos os dados (na primeira) e ocorridas na mediana quando se acrescenta dois dados em certas condições aos dados iniciais (na segunda). Em termos de resultados, salienta-se que, em média, se verificou uma taxa de sucesso de $72 \%$ por item, sendo essa taxa variável ao longo dos itens. Embora se trate de uma percentagem relativamente elevada de respostas corretas, constatou-se que os estudantes obtiveram as suas respostas através de estratégias pouco fiáveis em termos de garantir as respostas corretas, o que foi mais notório nos itens da segunda questão.
\end{abstract}

Palavras-chave: Estatística. Mediana e amplitude. Futuros professores. Primeiros anos escolares.

\section{Comprensión de Propiedades de la Mediana y Rango por Futuros Maestros}

\section{Resumen}

Este artículo estudia la comprensión de los estudiantes, futuros maestros, de las propiedades de las estadísticas mediana y amplitud total. En el estudio participaron 34 estudiantes que cursaban el 2. ${ }^{\circ}$ curso de la Licenciatura en Educación Básica en una universidad del norte de Portugal. Los estudiantes resolvieron varias preguntas, en el contexto del aula y con fines de evaluación formal, involucrando situaciones problema de Estadística Descriptiva, de las cuales presentaremos aquí solo dos. En estas preguntas, con dos ítems cada una, se evalúan los posibles cambios ocurridos en la mediana y en la amplitud total cuando se agrega un valor constante a todos los datos (en la primera) y en la mediana cuando se agregan dos datos bajo ciertas condiciones a los datos iniciales (en el segunda). En términos de resultados, cabe señalar que, en promedio, hubo una tasa de éxito del $72 \%$ por ítem, siendo esta tasa variable entre ítems. Si bien es un porcentaje relativamente alto de respuestas correctas, se encontró que los estudiantes obtuvieron sus respuestas a través de estrategias poco confiables en cuanto a garantizar las respuestas correctas, lo cual fue más evidente en los ítems de la segunda pregunta.

Palabras clave: Estadística. Mediana y amplitud. Futuros profesores. Primeros años escolares. 


\title{
Understanding of Median and Range Properties by Prospective Primary School Teachers
}

\begin{abstract}
This article studies the understanding of prospective primary school teachers of statistical properties of the median and range. The study involved 34 students who were attending the 2 nd year of the Basic Education Degree course at a university in northern Portugal. The students solved several questions, in the classroom context and for the purpose of formal assessment, involving problems of Descriptive Statistics, of which we will present here only two. In these questions, with two items each, the possible changes occurred in the median and in the range are evaluated when a constant value is added to all data (in the first problem) and in the median when two data are added under certain conditions to the initial data (in the second problem). In terms of results, it should be noted that, on average, there was a success rate of $72 \%$ per item, with this rate being variable across items. Although it is a relatively high percentage of correct answers, it was found that students obtained their answers through unreliable strategies in terms of guaranteeing the correct answers, which was more evident in the items of the second question. Keywords: Statistics. Median and range. Prospective teachers. First school years.
\end{abstract}

\section{Introdução}

Em Portugal, atualmente, o ensino da estatística faz parte de todos os programas escolares da disciplina de matemática, desde o início do ensino básico até ao final do ensino secundário (Ministério da Educação e Ciência, 2013, 2014). No ensino básico, sob a designação de organização e tratamento de dados, o tema inclui o estudo de estatística e probabilidades e foi incluído explicitamente, pela primeira vez, no $1 .^{\circ}$ ciclo do ensino básico (ciclo do $1 .^{\circ}$ ao $4 .^{\circ}$ ano de escolaridade) no programa de matemática de 2007 (Ministério da Educação, 2007). Em Portugal, o ensino básico desenvolve-se ao longo dos primeiros nove anos de escolaridade e estrutura-se em três ciclos: $1 .^{\circ}$ ciclo, do $1 .^{\circ}$ ano ao $4 .^{\circ}$ ano; $2 .^{\circ}$ ciclo, $5 .^{\circ}$ e $6 .^{\circ}$ anos; e $3 .^{\circ}$ ciclo, do 7. ${ }^{\circ}$ ano ao $9 .^{\circ}$ ano.

Ora o alargamento do ensino da estatística aos primeiros anos escolares coloca novos desafios à formação dos professores que ensinam nesse nível de escolaridade. Até há pouco tempo, estes professores, que são responsáveis pelo ensino das várias disciplinas do currículo escolar, não tinham qualquer formação de estatística durante a sua formação inicial. Só recentemente, com o alargamento do ensino da estatística aos primeiros anos escolares, é que estes professores passaram a ter alguma formação nessa área. Os participantes do presente estudo, futuros professores dos primeiros anos, frequentaram no ensino superior uma disciplina 
semestral de probabilidades e estatística, tal como geralmente acontece na generalidade das instituições de formação destes professores em Portugal.

Assim, importa saber se estes futuros professores são detentores de um conhecimento que lhes permita implementar um ensino de estatística de qualidade. Estudos prévios sobre gráficos, tabelas e medidas estatísticas (Fernandes, Batanero, \& Gea, 2019; Fernandes, Gonçalves, \& Barros, 2021) têm demonstrado que estes futuros professores dos primeiros anos revelam sentir dificuldades nesses conteúdos, seja ao nível dos variados objetos ou dos processos matemáticos implicados na resolução das tarefas (Godino, Batanero, \& Font, 2007).

E no caso das propriedades das medidas estatísticas, qual a compreensão que os estudantes, futuros professores dos primeiros anos escolares, desenvolveram no seu processo de aprendizagem? A aprendizagem de tais propriedades reveste-se de grande importância em termos formativos na medida em que contribui para o desenvolvimento do conhecimento concetual e procedimental dos estudantes (Hiebert \& Lefevre, 1986), além de permitir relacionar diferentes conteúdos estatísticos.

De entre as propriedades das várias medidas estatísticas, no presente estudo iremos estudar em que medida os futuros professores compreendem algumas dessas propriedades, relativas à mediana e à amplitude total. Mais concretamente, reconhecer e/ou justificar a consequência (alterar/manter) sobre a mediana e a amplitude total ao adicionar um valor constante a todos os dados e sobre a mediana ao acrescentar dois dados em certas condições.

Após a apresentação do estudo, na próxima secção desenvolve-se o enquadramento teórico, tendo por referência a fundamentação teórica e as investigações prévias, segue-se a secção de metodologia, em que se detalham mais os procedimentos de condução do estudo, e a secção de resultados, em que se apresentam, fundamentalmente, os resultados do estudo. Por fim, na secção de conclusão e discussão sintetizam-se e discutem-se os principais resultados do estudo realizado, neste último caso tendo por referência outros estudos, e extraem-se implicações para a formação destes futuros professores dos primeiros anos escolares.

\section{Enquadramento teórico}

Em geral, nesta secção discute-se a problemática do conhecimento dos futuros professores para ensinar estatística. Para tal, começa-se por discutir a fundamentação teórica do 
estudo e, de seguida, apresentam-se alguns resultados de estudos sobre o conhecimento de estudantes em diferentes propriedades das medidas de localização e de dispersão.

\section{Fundamentação teórica}

Desde o final do século passado tem-se assistido a um crescente interesse acerca do conhecimento que o professor deve possuir para poder ensinar os seus alunos, sendo o trabalho de Shulman (1986) pioneiro nesse domínio e seguindo-se muitos outros trabalhos, designadamente de Godino e colaboradores (e.g., Godino, 2002; Godino \& Batanero, 1994; Godino et al., 2007) e de Hill, Ball e Schilling (2008).

Em todos estes trabalhos se distinguem duas categorias principais de conhecimento que o professor precisa de desenvolver para poder ensinar: o conhecimento do conteúdo e o conhecimento pedagógico do conteúdo. A primeira refere-se ao conhecimento que a pessoa tem de mobilizar para resolver situações-problema, que não é necessariamente específico do professor, e a segunda é relativa ao conhecimento necessário para ensinar, portanto um conhecimento específico do ofício docente.

No presente estudo basearemos a fundamentação teórica no enfoque ontossemiótico do conhecimento e instrução matemática (Godino et al., 2007, 2017) pois esse modelo corresponde melhor aos propósitos do nosso estudo, concretamente no que respeita aos significados do conhecimento dos futuros professores, além de nos fornecer diferentes ferramentas concetuais e metodológicas para analisar as suas respostas.

O enfoque ontossemiótico assume que o conhecimento matemático (e não só) emerge das práticas operativas e discursivas que o sujeito realiza ao resolver uma amostra representativa de situações-problema referentes a um dado conteúdo matemático. Ora, segundo Godino (2002), em tais práticas de resolução de problemas intervém uma diversidade de objetos matemáticos primários, como sejam:

- As situações-problema, que são ações que induzem uma atividade matemática (aplicações extramatemáticas, exercícios, problemas);

- Linguagens, que se utilizam para representar os dados de um problema, as operações que se efetuam, os objetos matemáticos que se usam e a solução encontrada em seus diversos registos (termos, expressões, notações, gráficos); 
- Conceitos/definições, que são formulações introduzidas mediante definições e descrições, as quais constituem práticas realizadas para resolver um problema matemático com uso implícito ou explícito de objetos matemáticos, que o aluno tem de lembrar e aplicar;

- Propriedades/proposições, que são enunciados sobre relações ou propriedades dos conceitos que se utilizam para resolver problemas matemáticos;

- Procedimentos, que se referem a algoritmos, operações, técnicas de cálculo que os alunos aplicam para a resolução do problema; e

- Argumentos, que são enunciados usados nas respostas para validar ou explicar as proposições e procedimentos ou a solução dos problemas, que podem ser dedutivos, indutivos, formais ou informais.

$\mathrm{Na}$ resolução de uma situação-problema intervêm os objetos matemáticos primários, antes referidos, relacionando-se de formas diversas e constituindo as assim designadas configurações de objetos, que podem ser de tipo epistémico (se são próprias de uma instituição) ou cognitivo (se são específicas de um estudante, no nosso caso um futuro professor) (Godino et al., 2007).

O nosso propósito é investigar esta dualidade do conhecimento, institucional versus pessoal, a partir das respostas dadas pelos estudantes às questões sobre as propriedades das estatísticas implicadas. Para tal, consideramos o significado de um ponto de vista institucional (estabelecido pelos estatísticos) e de um ponto de vista pessoal (relativo a cada um dos futuros professores dos primeiros anos escolares) (Godino \& Batanero, 1994).

Além da dualidade institucional-pessoal, antes referida, no enfoque ontossemiótico reconhecem-se outras dualidades (Godino et al., 2007), das quais é, também, relevante para o nosso estudo a dualidade expressão-conteúdo, que permite confrontar os significados dos objetos que intervêm nas funções semióticas (entendidas como correspondências estabelecidas por uma pessoa ou instituição entre um antecedente, expressão, e um consequente, conteúdo) com os significados institucionais de referência. No processo de comparação, a verificação de discrepâncias entre esses significados, ou seja, entre os significados institucional e pessoal (Godino \& Batanero, 1994), conduz à identificação de conflitos semióticos.

Tendo em conta a dualidade do conhecimento, institucional versus pessoal, podemos concluir que a finalidade do ensino é fazer coincidir, o mais possível, o conhecimento pessoal 
com o conhecimento institucional. Neste contexto, Godino e Batanero (1994) definem que a compreensão de um conteúdo por um sujeito corresponde à parte do seu conhecimento pessoal que é comum com o conhecimento institucional.

\section{Investigação prévia}

Em geral, os futuros professores dos primeiros anos revelam dificuldades em variados conteúdos de estatística, incluindo nas medidas de tendência central (Jacobbe, 2011). Por exemplo, Fernandes e Barros (2005) verificaram que futuros professores dos primeiros anos sentiram dificuldades em situações em era requerida a inversão do algoritmo da média, que se agravaram no caso do significado e interpretação da mediana e média. Também Batanero, Godino e Navas (1997) analisaram as respostas de futuros professores do ensino primário a um questionário escrito, que incluía quatro itens sobre distintos aspetos interpretativos da média aritmética, em que os resultados mostraram a existência de erros concetuais e dificuldades de aplicação dos conhecimentos sobre as medidas de tendência central. Entre os mais frequentes, verificou-se que $40 \%$ da amostra não descartou os valores atípicos para calcular la media, 30\% sobrevalorizou a dispersão dos dados e $60 \%$ não conhecia a relação entre média, mediana e moda em distribuições simétricas. Estas dificuldades confirmaram-se num estudo posterior de Estrada, Batanero e Fortuny (2004), envolvendo também futuros professores do ensino primário.

No caso das propriedades das medidas estatísticas de tendência central e de dispersão, que são o foco do presente estudo, não encontrámos estudos envolvendo futuros professores dos primeiros anos, donde, seguidamente, vamos referi-nos a alguns estudos tendo por participantes outros alunos.

Strauss e Bichler (1988) realizaram uma investigação, envolvendo alunos de idades entre os 8 e os 14 anos, para estudarem o reconhecimento e a aplicação das seguintes propriedades da média: a) A média situa-se entre os valores extremos da distribuição; b) A soma dos desvios dos dados em relação à média é zero; c) A média é influenciada pelo valor de cada um dos dados; d) A média não é necessariamente igual a um dos valores do conjunto de dados; e) O valor da média pode não ser inteiro; f) Os dados com valor nulo influenciam o cálculo da média e g) A média é um "representante" do conjunto de dados a que diz respeito. Neste estudo foram utilizadas tarefas diversas, incluindo a consideração do tipo de dados discretos e contínuos. 
Em termos de resultados, foi observada uma melhoria sistemática da compreensão com a idade e, para uma mesma idade dos alunos, o grau de compreensão das propriedades foi variável, oferecendo menos dificuldades as propriedades a), c) e d) e revelando-se mais difíceis as propriedades b), f) e g). Já a análise dos raciocínios dos alunos mostrou que as dificuldades dos alunos aumentaram com o maior grau de complexidade das propriedades e a aplicação das propriedades a conjuntos de dados discretos ou contínuos esteve na origem de níveis de complexidade distintos, tendo-se revelado mais difícil para os alunos os dados de tipo contínuo.

Leon e Zawojewski (1991) conduziram entrevistas a crianças dos 8 aos 14 anos para estudar a influência da idade sobre as propriedades da média investigadas por Strauss e Bichler (1988), tendo encontrado uma importante influência da idade na compreensão da média e que a contextualização das tarefas facilita muito a sua resolução. Contudo, propriedades como b) A soma dos desvios dos dados em relação à média é zero, f) Os dados com valor nulo influenciam o cálculo da média ou g) A média é um "representante" do conjunto de dados a que diz respeito continuaram a ser muito abstratas para um número importante de alunos de 14 anos.

As dificuldades dos alunos, segundo Leon e Zawojewski (1991), devem-se a que as duas primeiras propriedades envolvem a ideia de distribuição de dados e sua relação com a média, que os alunos consideram fácil de compreender, enquanto as duas últimas lidam com a interpretação implícita da média. Adicionalmente, os autores também notaram que as duas primeiras propriedades eram baseadas em computação, embora os alunos não as pudessem verificar aplicando números e operações, enquanto nas duas últimas isso não acontece.

Boaventura e Fernandes (2004) questionaram alunos do 12..$^{\circ}$ ano sobre propriedades da média e da mediana, portanto alunos de um nível de escolaridade mais avançado. No caso da média inquiriram-se os alunos sobre a verdade ou falsidade das propriedades: a) A média de um conjunto de dados nunca é igual a um dos dados; b) A média de um conjunto de dados é sempre um valor compreendido entre o menor e o maior dos dados; c) Se acrescentarmos o valor 0 (zero) ao conjunto de dados, o valor da média não se altera; d) Se acrescentarmos o valor da média ao conjunto de dados, o valor da média não se altera e e) Dois conjuntos de dados diferentes podem ter a mesma média. Nestas propriedades as percentagens de respostas corretas variaram entre 47,5\% e 93,9\%, com uma média de 75,2\% de acerto nos cinco itens, destacandose maiores dificuldades dos alunos nos itens c) e d). Muitos alunos acreditam que acrescentar o valor 0 (zero) não altera o valor da média, porque o veem como elemento neutro, e mais alunos 
sentiram ainda dificuldade em concluir que a média não se altera quando se acrescenta um dado com o valor da média.

No caso da propriedade acrescentar o valor 0 (zero) ao conjunto de dados, Cazorla (2003) constatou que a maioria dos estudantes universitários $(79,5 \%)$ que participaram no seu estudo, focado na compreensão do conceito de média, respondeu corretamente, sendo que o erro mais frequente consistiu em ignorar o novo dado e calcular novamente a média dos números dados.

Ainda no estudo de Boaventura e Fernandes (2004), dada a média dos vencimentos dos trabalhadores de uma empresa, inquiriram-se os alunos sobre o novo valor da média quando o vencimento cada trabalhador foi aumentado a) de 25 euros e b) em $5 \%$. O desempenho dos alunos foi semelhante nestes dois itens, tendo-se verificado que 59,7\% e 58,0\% dos alunos responderam corretamente, respetivamente, ao item a) e ao item b). Nos dois itens, os erros dos alunos resultaram, principalmente, do uso de fórmulas incorretas, de erros de cálculo e/ou de leitura incorreta de dados do enunciado e da dificuldade de trabalhar com percentagens. Nas respostas erradas de ambos os itens, salienta-se ainda a ausência de sentido crítico dos alunos quando apresentaram um novo valor da média muito superior ou muito inferior ao valor dado.

No caso da mediana, questionaram-se os alunos sobre se o valor da mediana diminui, aumenta ou mantém-se quando: a) Se acrescenta um dado inferior a todos os outros; b) Se acrescenta um dado com o valor da mediana; c) Se acrescenta um dado com valor superior ao da mediana; d) Se acrescenta dois dados, um dado inferior a todos os outros e outro superior a todos os outros. A percentagem de respostas corretas variou entre $65,7 \%$ e $82,3 \%$, com uma média $72,9 \%$ de acerto na globalidade dos quatro itens. Verificou-se, ainda, que as dificuldades dos alunos foram um pouco superiores no caso de se acrescentar um dado inferior a todos os outros, um dado igual à mediana ou um dado superior à mediana.

Num estudo mais recente, Freitas, Figueiredo, Silva e Miranda (2018) realizaram um estudo com alunos do $8 .^{\circ}$ ano sobre possíveis alterações da mediana e do diagrama de extremos e quartis decorrentes de alterações dos dados, portanto envolvendo uma abordagem analítica e uma abordagem gráfica. No caso da abordagem analítica, começava-se por pedir aos alunos para determinarem os quartis de um conjunto de dados, após o que eram questionados sobre o que acontece à mediana quando se 1) substitui o valor mínimo por um valor inferior, 2) acrescenta um valor superior a todos os outros e 3 ) acrescenta um valor inferior a todos os outros. Em todos 
estes três itens obtiveram-se percentagens de respostas corretas de $70 \%, 85 \%$ e $83 \%$, respetivamente, o que revela um bom desempenho dos alunos.

$\mathrm{Na}$ abordagem gráfica, começava-se por pedir aos alunos para indicarem ou determinarem, a partir do digrama de extremos e quartis dado, os quartis e o intervalo interquartil. De seguida, questionavam-se os alunos sobre se o diagrama de extremos e quartis se mantém inalterado, se pode sofrer alterações ou se sofre necessariamente alterações quando se substitui um dado por um outro diferente, designadamente 1) substituir um valor do intervalo ] $Q_{3}$, máx [ pelo valor máx, 2) substituir um valor do intervalo ] mín, $Q_{1}$ [ por outro valor desse intervalo, 3) substituir o valor de $Q_{2}$ pelo valor de $\left.Q_{1}, 4\right)$ alterar o valor mín, 5) alterar o valor máx e 6) substituir um valor do intervalo $] Q_{2}, Q_{3}$ [ por outro valor desse intervalo. Os alunos revelaram mais dificuldades nesta tarefa do que na anterior, tendo-se obtido percentagens de respostas corretas de $42 \%, 58 \%, 36 \%, 42 \%, 40 \%$ e 49\%, respetivamente em cada um dos itens.

Face aos resultados obtidos, os autores estudaram o tipo de erros cometidos pelos alunos, tendo concluído que as dificuldades se devem a um "domínio deficiente" do conhecimento no caso da mediana, enquanto no caso do diagrama de extremos e quartis se devem "a incapacidade dos alunos de extrapolar informação visual e espacial" (p. 124). Além disso, os autores concluíram que não existe qualquer associação entre os tipos de respostas dadas pelos alunos na tarefa da mediana e na tarefa do diagrama de extremos e quartis. Portanto, quando se altera ou acrescenta um dado, conclui-se que não existe influência entre identificar/determinar a mediana a partir de uma coleção de dados e a partir da descrição gráfica de um diagrama de extremos e quartis.

No caso do desvio padrão, delMas e Liu (2005) conduziram um estudo com estudantes universitários sobre a compreensão do conceito de desvio padrão. Depois de concluído um processo formativo, em que os estudantes foram questionados sobre a reorganização dos dados num histograma de modo a cumprir um critério especificado (e.g., obter o maior desvio padrão possível), foi aplicado um teste com 10 itens, cada um constituído por dois histogramas, dos quais se conhecia média dos dois e o desvio padrão de apenas um, inquirindo-se o estudante sobre se o desvio padrão do outro era menor, maior ou igual do que o valor do desvio padrão dado. Num desses itens, exatamente o primeiro, o segundo histograma resulta de uma deslocação horizontal do primeiro, o que equivale a adicionar um valor constante a todos os 
dados. Neste item, todos estudantes responderam corretamente ao afirmarem que o valor do desvio padrão se mantém.

\section{Metodologia}

Tal como foi referido antes, neste artigo estuda-se a compreensão de estudantes, futuros professores dos primeiros anos escolares, de propriedades das estatísticas mediana e amplitude total, quando se adiciona um valor constante a todos os dados e quando se acrescenta dois dados em certas condições aos dados iniciais.

Participaram no estudo 34 estudantes do 2. ${ }^{\circ}$ ano do curso de Licenciatura em Educação Básica, de uma universidade do norte de Portugal. Estes estudantes, futuros professores dos primeiros anos, tinham uma formação matemática muito variada à entrada na universidade, que ia desde a formação matemática obtida no final do $9 .^{\circ}$ ano (final da escolaridade básica) ou em cursos do nível de ensino secundário, como sejam cursos profissionais, cursos humanísticos e cursos científico-tecnológicos.

Os dados usados no presente estudo foram obtidos através das respostas escritas dadas pelos estudantes a um questionário de avaliação formal sobre estatística descritiva. O questionário era constituído por várias questões, das quais estudamos aqui apenas duas, cujos enunciados são apresentados na Figura 1.

Figura 1. Tarefa proposta aos estudantes.

1. Tem-se um conjunto de $n$ dados numéricos e, seguidamente, adicionou-se o valor 15 a cada um dos dados. Quando se adiciona o valor 15 a cada um dos dados, o que acontece a cada uma das seguintes estatísticas:
a) Mediana;
b) Amplitude total.

2. Considere que se tem um conjunto de $n$ dados numéricos, com $n>4$, todos distintos. Relativamente a cada caso seguinte, justificando, diga se o valor da mediana se mantém, diminui ou aumenta, quando se:

a) Acrescenta dois dados, um menor e outro maior do que todos os outros;

b) Acrescenta dois dados, um igual à mediana e outro maior do que todos os outros.

Fonte: Elaboração do autor.

Constata-se, assim, que na primeira questão, com dois itens, se pede aos estudantes que definam as possíveis alterações da mediana e da amplitude total quando se aumentam todos os 
dados do valor 15. Esperava-se, portanto, que os estudantes concluíssem que a mediana também vem acrescida do valor 15 e que amplitude total se mantém inalterada.

$\mathrm{Na}$ segunda questão, também com dois itens, questionam-se os estudantes sobre as possíveis alterações da mediana quando se acrescenta dois dados, um menor e outro maior do que todos os outros ou um igual à mediana e outro maior do que todos os outros. Agora, esperava-se que os estudantes concluíssem que a mediana se mantém inalterada quando se acrescenta dois dados que são os novos extremos e que se mantém ou aumenta no outro caso. Deve notar-se, desde já, que a obtenção da resposta correta no primeiro item é mais óbvia e intuitiva do que no segundo item, que envolve um grau de desafio mais elevado para os estudantes.

Por último, em relação ao tratamento e análise de dados, estudaram-se as respostas apresentadas pelos estudantes, relativamente à correção e incorreção dessas respostas e às ideias subjacentes a essas respostas. Em ambos os casos, determinaram-se frequências dos tipos de respostas (corretas e incorretas) e das ideias subjacentes a tais respostas, tendo-se usado uma tabela para resumir a informação dos tipos de respostas. Adicionalmente, tendo em vista proporcionar uma melhor compreensão das respostas dos estudantes e da análise realizada, serão ainda apresentados alguns exemplos de respostas dos estudantes, identificados pela letra $E$ (abreviatura de estudante) seguida do número que lhe foi atribuído (de 1 a 34).

\section{Resultados}

$\mathrm{Na}$ análise dos resultados do estudo exploram-se as resoluções dos estudantes da tarefa proposta numa dupla perspetiva: 1) estudo das respostas, relativo à classificação das respostas em corretas e incorretas; 2) estudo das estratégias, relativo ao processo de obtenção das respostas.

\section{Respostas}

Em termos de resolução da tarefa, esperava-se que os estudantes apresentassem, em cada um dos itens, as respostas seguintes:

Em 1a), depois de ordenados os dados e adicionado o valor 15 a cada um deles, a mediana vem aumentada do valor 15 pois o dado central vem aumentado de 15 (no caso de $n$ 
ímpar) ou os dois dados centrais vêm aumentados de 15 e, portanto, a sua semissoma também vem aumentada de 15 (no caso de $n$ par);

Em 1b), a amplitude total mantém-se (ou não se altera) uma vez que tanto o valor máximo como o valor mínimo dos dados iniciais vêm aumentados de 15, e os dois valores 15 anulam-se ao calcular a diferença correspondente à amplitude total, ou seja, $h=($ má $x+15)-$ $($ mín +15$)=$ máx $-\operatorname{mín}$;

Em 2a), depois de ordenados os dados, todos distintos, a mediana mantém-se porque ao acrescentar-se um dado menor e outro maior do que todos os outros, o dado central (no caso de $n$ ímpar) ou os dois dados centrais (no caso de $n$ par) mantêm-se e, portanto, também a mediana se mantém;

Em 2b), depois de ordenados os dados, todos distintos, a mediana mantém-se quando $n$ é ímpar pois ao acrescentarem-se os dois dados (um igual à mediana e outro maior do que todos os outros) continuamos a ter um número ímpar de dados, donde a mediana é o valor central, coincidindo com o valor da mediana anterior; a mediana aumenta quando $n$ é par pois ao acrescentarem-se os dois dados continuamos a ter um número par de dados, donde a mediana é a semissoma dos dois valores centrais, em que um deles é a mediana dos dados iniciais e o outro é o dado imediatamente superior, dado esse que é maior do que a mediana inicial.

$\mathrm{Na}$ Tabela 1, apresentada a seguir, estão registadas as frequências de estudantes segundo os tipos de resposta correta e incorreta e de não resposta em cada um dos quatro itens da tarefa.

Tabela 1

Frequência (em \%) de estudantes segundo os tipos de resposta e não resposta nos itens

\begin{tabular}{lcccc}
\multirow{2}{*}{ Tipo de resposta } & \multicolumn{4}{c}{ Itens } \\
\cline { 2 - 5 } & 1a) & 1b) & 2a) & 2b) \\
\hline Correta & $30(88)$ & $26(76)$ & $34(100)$ & $8(24)$ \\
Incorreta & $4(12)$ & $7(21)$ & - & $26(76)$ \\
Não resposta & - & $1(3)$ & - & - \\
\hline
\end{tabular}

Fonte: Elaboração do autor.

Por observação da Tabela 1 conclui-se que cerca de três ou mais em cada quatro estudantes apresentaram a resposta correta aos três primeiros itens, enquanto apenas cerca de um em cada quatro responderam corretamente ao último item. 
Entre os dois primeiros itens, 1a) e 1b), em que se questionava sobre a consequência na mediana e na amplitude total ao adicionar um valor constante a todos os dados, verifica-se que os estudantes tiveram um pior desempenho no item $1 \mathrm{~b}$ ), que envolve a estatística da amplitude total dos dados. Embora se possa pensar que os dois itens são semelhantes no grau de desafio que envolvem, a exploração do item 1a) é mais habitual nas aulas de Estatística do que o item 1b), além de que ele mantém alguma analogia com o correspondente item que envolve o conceito de média aritmética.

Já no caso dos dois últimos itens, 2a) e 2b), em que se questionava sobre a consequência de acrescentar dois dados em certas condições à série de dados fornecidos, verifica-se que o desempenho dos estudantes foi claramente inferior no item $2 b$ ), em que se acrescentava dois novos dados, um igual à mediana e outro maior do que todos os outros.

No caso do item 2a), no estudo de Boaventura e Fernandes (2004), os alunos do 12. ano, participantes do estudo, obtiveram também um bom desempenho no item $2 a)(82,3 \%)$ e os alunos do $8 .^{\circ}$ ano, que participaram no estudo de Freitas et al. (2018), também revelaram um bom desempenho nos itens em que se acrescentava apenas um dado, maior do que todos os outros $(85 \%)$ ou menor do que todos os outros $(83 \%)$.

O pior desempenho dos estudantes no item 2 b), o qual se destaca de entre todos os itens, certamente tem origem no facto de a resposta correta neste item ser diferente consoante o número de dados, $n$, é ímpar ou par. No item 2a) isso não acontece, sendo a resposta correta a mesma quer o número de dados, $n$, seja ímpar ou seja par. Realmente, essa particularidade do item $2 \mathrm{~b}$ ) teve um impacto muito forte no desempenho dos estudantes, observando-se uma percentagem de respostas corretas muito baixa (24\%), muito inferior às percentagens obtidas nos vários itens dos estudos de Strauss e Bichler (1988), Boaventura e Fernandes (2004) e de Freitas et al. (2018).

Contudo, a análise anterior, centrada no tipo de resposta correta ou incorreta, não capta todo o pensamento desenvolvido pelos estudantes no processo de resolução da tarefa. Portanto, para além do resultado final (resposta correta e incorreta), iremos seguidamente analisar as estratégias a que os estudantes recorreram para obter as suas respostas. A descrição das estratégias usadas pelos estudantes permite identificar e compreender as ideias por eles mobilizadas no processo de resolução dos vários itens da tarefa. 
Ora uma tal análise das estratégias requer que os estudantes explicitem, com o maior detalhe possível, todo o processo que os conduziu à resposta apresentada, tal como aconteceu nos itens $2 \mathrm{a}$ ) e $2 \mathrm{~b}$ ), em que se pedia que justificassem as suas respostas. Diferentemente, nos itens 1a) e 1b) não se pedia qualquer justificação, pelo que essa análise assumirá um caráter esporádico, dependente da iniciativa dos estudantes descreverem, de alguma maneira, o processo de resolução que usaram.

\section{Estratégias}

Tal como referimos antes, nos itens 1a) e 1b) não foi requerido dos estudantes a justificação das suas respostas, tendo-se verificado que apenas quatro apresentaram justificações em 1a) e 12 em 1b), sendo todas essas justificações relativas a respostas corretas. Em consequência desse reduzido número de justificações, as estratégias identificadas, que se apresentam a seguir, são meramente indicativas das formas de pensar dos estudantes nestes itens. Já nos itens 2a) e 2b) foi solicitado aos estudantes que justificassem as suas respostas, verificando-se que apenas dois deles não apresentaram qualquer explicação das suas respostas em cada um dos itens. Donde podemos considerar que nestes itens as estratégias identificadas são representativas das ideias mobilizadas pelos estudantes.

Tabela 2

Frequência (em \%) de estudantes segundo as estratégias usadas para obter as respostas

\begin{tabular}{lcccc} 
Estratégia & \multicolumn{3}{c}{ Itens } \\
\cline { 2 - 5 } & 1a) & 1b) & 2a) & 2b) \\
\hline Analisar exemplos & $1(3)$ & $4(12)$ & $14(41)$ & $27(79)$ \\
Foco no valor central e/ou valores centrais & & & $10(29)$ \\
Foco nos valores extremos & & $8(24)$ & \\
Definição da amplitude total & $7(20)$ & & \\
Manter a alteração dos dados & $3(9)$ & $1(3)$ & & $4(12)$ \\
Distinguir dois casos para a mediana & & & & $1(3)$ \\
Ininteligível & & & & $32(94)$ \\
Total & $4(12)$ & $12(35)$ & $32(94)$ & 3 \\
\hline
\end{tabular}

Fonte: Elaboração do autor. 
$\mathrm{Na}$ Tabela 2 apresenta-se a síntese das estratégias adotadas pelos estudantes para obterem as suas respostas em cada um dos itens. As percentagens da tabela foram calculadas em relação ao número total de participantes do estudo, isto é, 34.

De entre as várias estratégias, em termos de frequência, salienta-se a estratégia de análise de exemplos, que foi adotada em todos os itens. Segue-se o foco no valor central e/ou valores centrais para determinação da mediana (no item 2a)), o foco nos valores extremos para determinação da mediana (no item 2a)) e a definição da amplitude total (no item 1b)). Por último, com menor frequência, os estudantes afirmaram que as alterações verificadas nos dados se repetiam nas estatísticas (nos itens 1a) e 1b)) e distinguiram dois casos para a mediana (no item 2b)). $\mathrm{Na}$ continuação, detalham-se mais as estratégias referidas em cada item, apresentando-se também alguns exemplos de justificações apresentadas pelos estudantes tendo em vista clarificar e compreender melhor as suas ideias.

Em três das justificações apresentadas no item 1a), os estudantes apenas referiram que o aumento do valor 15 nos dados se repercutiria no aumento do mesmo valor da mediana, não explicando qualquer relação entre o aumento dos dados e o aumento da mediana. Deste modo, os estudantes afirmaram que a alteração a que foram submetidos os dados se repercutiu (manteve-se) na mediana. Já a outra justificação, apresentada por um estudante apenas, baseouse no estudo de um exemplo, como se mostra na resolução do respetivo estudante.

A mediana (corresponde ao valor central, divide a distribuição em 2 partes iguais) vai aumentar, visto que a cada um dos dados vai ser adicionado o valor 15 . Todos os dados vão ficar superiores ao que eram inicialmente, logo o valor da mediana vai também aumentar.

Ex: se os dados iniciais forem 1, 2, 3, 4 e 5. A mediana correspondente vai ser 3 .

Depois de adicionar o valor 15 fica: 16, 17, 18, 19 e 20. Logo neste caso a mediana vai ser 18 . E 18 é superior a 3 .

Se a mediana inicial é 3 , então depois de adicionarmos o valor 15 , fica $3+15$, logo a nova mediana passaria a ser 18 . $\left(E_{18}\right)$

Pela resposta do estudante constata-se que ele começa por concluir que o aumento dos dados no valor 15 acarreta um aumento da mediana, sem quantificar esse aumento. De seguida estuda uma série de 5 dados, portanto com um número ímpar de dados, e conclui que esse aumento também é de 15. Repare-se que o facto de o estudante considerar apenas o caso ímpar não levanta problema pois a mesma conclusão se obtém quando a série de dados é par. 
No item 1b) os estudantes apresentam uma maior diversidade de justificações, sendo que a maior parte deles (7) recorre à definição de amplitude total para obter a sua resposta, como se exemplifica com a resolução do estudante seguinte:

b) A amplitude total permanece igual. $h=($ má $x+15)-(m i ́ n+15)$. Permanece igual, pois tanto o máximo como o mínimo vão aumentar 15 pontos, assim a sua diferença continua a mesma $\left(E_{9}\right)$.

Embora não efetue os cálculos, o estudante diz por palavras que a diferença entre dois valores não se altera quando se adiciona o mesmo valor a ambos os operandos, isto é, ao aditivo e ao subtrativo.

$\mathrm{Na}$ estratégia seguinte mais adotada, quatro estudantes recorreram a um exemplo para concluírem que a amplitude total dos dados se mantinha, como se exemplifica com a resolução do seguinte estudante:

A amplitude continuará a ser a mesma porque ao valor maior e ao valor menor será adicionado o mesmo valor, ou seja, se o valor maior do conjunto de dados $n$ for 5 e o valor menor for 1, então a amplitude será $5-1=4$. Acrescentando o valor 15 , a amplitude seria $20-16=4 .\left(E_{24}\right)$.

Neste exemplo verificamos que o estudante indicou o valor máximo e o valor mínimo de um conjunto de dados que arbitrou, mas que não indica, e de seguida determinou a amplitude. Finalmente, determinou o valor da nova amplitude, relativa aos dados que resultaram da soma do valor 15 e concluiu que não se alterou o valor da amplitude determinada antes. Implicitamente, o estudante assume que ao adicionar 15 ao valor máximo e ao valor mínimo inicial se obtém o valor máximo e o valor mínimo dos novos dados.

Por fim, à semelhança do que se verificou no item 1a), um estudante apenas referiu que o aumento dos dados no valor 15 não alterava o valor da amplitude total, como se ilustra na seguinte resolução:

A amplitude mantém-se visto que todos os dados numéricos aumentam na mesma proporção $\left(E_{15}\right)$.

Claro que, diferentemente, o aumento dos dados no mesmo valor repercutiu-se no aumento da mediana de igual valor (no item 1a)) e na permanência da amplitude (no item b)).

Nos itens 2a) e 2b), dado um conjunto de dados todos distintos, questionaram-se os estudantes sobre se a mediana se mantém, diminui ou aumenta quando se acrescenta dois dados em certas condições. Nestes dois itens apenas dois estudantes não apresentaram qualquer 
justificação da sua resposta, o que permite obter uma melhor representatividade das ideias dos estudantes.

Conforme foi referido antes, no item 2a) todos os estudantes deram a resposta correta (ver Tabela 1) e as justificações dadas deram origem a três tipos de estratégias. A estratégia adotada por mais estudantes (14) consistiu em apresentar e analisar um exemplo. Desses exemplos, a maioria (12) envolvia um total de dados ímpar e muito poucos (2) um total de dados par. Adicionalmente, dos exemplos envolvendo um número ímpar de dados, alguns (5) revelam uma tendência, embora incipiente, de generalização, como se ilustra na resolução apresentada pelo seguinte estudante:

a) Exemplo: $\underline{6} 789 \underline{10}$. A mediana não se altera porque os dados que são acrescentados são os extremos, logo não interfere. $\left(E_{32}\right)$

O estudante em questão começa por considerar uma série de três dados, não respeitando o mínimo de cinco dados decorrente do enunciado, acrescenta os dois novos dados e conclui que a mediana se mantém. Seguidamente, ele refere que os dois dados acrescentados, porque são os extremos, não interferem com a mediana, o que parece ir além do exemplo apresentado e, portanto, constituir um passo para a generalização.

Ora, a ideia de que os extremos não interferem com a mediana constitui uma constatação importante da natureza da mediana em relação a outras medidas estatísticas (como seja a média), assumindo-se como uma medida estatística robusta. Além disso, nesta situação-problema, a exploração de um exemplo com um número ímpar ou par de dados conduz ao mesmo tipo de resposta, mais concretamente à resposta correta. Ora, certamente, este facto também terá contribuído para que todos os estudantes tivessem respondido corretamente ao item.

Bastantes estudantes (10) focaram-se no processo de determinação da mediana, referindo especificamente que na determinação da mediana interessa o valor central ou os dois valores centrais, consoante o número de dados é ímpar ou par, respetivamente. Seguidamente apresentase a resolução de um estudante que adotou esta estratégia.

a) 2 dados. A mediana mantém-se visto que os dados inseridos são os extremos do conjunto de dados, isto é, temos um valor mínimo e um máximo logo a mediana não se altera, pois, sendo o dado do meio do conjunto, ou o resultado da soma dos dois dados do meio dividida por dois, não se altera quando inserido um valor mínimo e outro máximo. $\left(E_{30}\right)$ 
Este estudante, conjuntamente com outro, foram os dois que se referiram, simultaneamente, à mediana como sendo o "valor central" ou a semissoma dos "dois valores centrais", o que indicia que tiveram em conta o caso de o número total de dados ser ímpar ou ser par, enquanto, dos restantes, quatro se referiram apenas ao "valor central" e outros quatro apenas aos "valores centrais".

Ora, a alusão ao "valor central" parece significar que o estudante se centrou num conjunto de dados ímpar, enquanto a alusão aos "valores centrais" parece significar que ele se focou num conjunto de dados par. Embora nesta situação-problema a consideração dos casos de $n$ ímpar e $n$ par não seja imprescindível para a obtenção da resposta correta, como já foi antes referido, trata-se, todavia, de uma estratégia com limitações, podendo mesmo revelar-se falível em outras situações-problema, como veremos no item $2 b$ ), onde essa exigência se repercutiu muito negativamente no desempenho dos estudantes (ver Tabela 1).

Por fim, um pouco menos estudantes (8) argumentaram que acrescentar um dado maior e outro menor do que todos os outros, que constituem os valores extremos do conjunto de dados aumentado, não altera o valor da mediana. A título de exemplo desta estratégia, apresenta-se a resolução do estudante $E_{6}$ : "O valor da mediana mantém-se porque se temos um valor menor e outro maior não vai alterar o valor da mediana". Comparativamente com as duas estratégias anteriores, esta não está tão centrada no significado da mediana pois apenas se refere que a mediana se mantém, enquanto as duas outras estratégias envolvem explicitamente e implicitamente o processo de determinação da mediana, respetivamente, no caso dos exemplos e do "valor central" e/ou "valores centrais".

Por último, no item 2b) a obtenção da resposta correta requeria que o estudante considerasse, separadamente, as hipóteses de o número total de dados ser ímpar ou par, pois se for ímpar, a mediana mantém-se e se for par, a mediana aumenta. É exatamente essa exigência que está na origem da grande redução do número de respostas corretas neste item (8 corretas e 26 incorretas). Este resultado confirma a importância de os estudantes analisarem as duas hipóteses, quando $n$ é ímpar e quando $n$ é par, pois, em situações-problema deste tipo, o estudo apenas do caso ímpar ou apenas do caso par levará o estudante a responder incorretamente.

Em todas as respostas corretas (8) os estudantes apresentaram um exemplo para $n$ ímpar e outro exemplo para $n$ par. Assim, os estudantes determinaram a mediana em cada exemplo e reconheceram, de seguida, os dois casos, como se mostra na resposta apresentada na Figura 2. 
Figura 2. Resolução do item 2b) pelo estudante $E_{25}$.

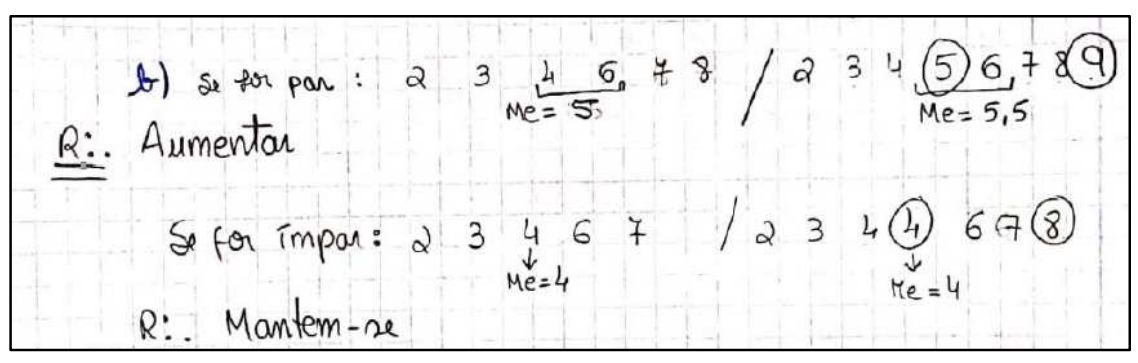

Observa-se que o estudante partiu de conjuntos com seis e cinco dados distintos, determina as respetivas medianas, acrescenta essas medianas aos conjuntos iniciais bem como um dado maior do que todos os outros e conclui que a mediana aumenta quando $n$ é par e mantém-se quando $n$ é ímpar. Embora este e os outros estudantes tenham chegado às suas respostas a partir da análise de exemplos particulares, tal não invalida o tipo de resposta (correta) a que chegaram.

No caso das respostas incorretas, muitos estudantes (16) exploraram apenas um exemplo, quase sempre com um número total de dados ímpar (apenas um recorreu a um número total de dados par). Seguidamente apresenta-se a resolução de um estudante que seguiu esta estratégia.

A mediana mantém-se, visto que vamos acrescentar dois valores e um deles vai para um extremo dos números ordenados e o outro número que se acrescentou é igual à mediana. Por exemplo, temos os valores 7911 e vamos acrescentar o 9 e 12, ficamos então com 79911 12, logo a mediana é 9 e mantém-se. $\left(E_{4}\right)$

Verifica-se que o estudante começou por arbitrar um conjunto de três dados, não cumprindo uma das condições do enunciado $(n>4)$, determinou a mediana e acrescentou-a aos três dados iniciais bem como um valor maior do que todos os outros e, por fim, determinou a mediana do novo conjunto de dados. Desse modo, o estudante obteve a conclusão correta para o caso de $n$ ímpar, mas não estudou o caso de $n$ par, que conduziria à conclusão de que a mediana aumenta.

Constatou-se, ainda, que poucos estudantes (3) recorreram a dois conjuntos de dados, um com um número de dados ímpar e outro com um número de dados par, mas chegaram a uma conclusão incorreta porque cometeram erros no processo de determinação das respetivas medianas. Na Figura 3 apresenta-se um exemplo em que isso acontece. 
Figura 3. Resolução do item 2b) pelo estudante $E_{9}$.



Na resolução do estudante constata-se que ele arbitrou um conjunto de seis dados (no caso de $n$ par) e determinou corretamente a mediana, obtendo o valor 4,5 . Contudo, de seguida, acrescenta o valor 4 ao conjunto de dados inicial e não o valor da mediana, como era requerido, concluindo, assim, que a mediana se mantém.

Dos restantes estudantes, todos eles apresentando respostas incorretas, quatro afirmaram conclusões incorretas para $n$ ímpar e/ou para $n$ par sem darem qualquer explicação e um apresentou uma justificação ininteligível. Por exemplo, o estudante $E_{7}$ afirmou que "Se o número for ímpar, a mediana mantém-se. Se o número for par também se mantém" e o estudante $E_{8}$ afirmou que "Depende. Se um dos dados for par, a mediana diminui, se for ímpar a mediana mantém-se".

\section{Conclusão e discussão}

No caso da amplitude total, avaliada apenas através do item 1b), aproximadamente três em cada quatro estudantes responderam corretamente que a amplitude não se altera quando se adiciona a todos os dados o valor 15. Dos poucos estudantes que justificaram as suas respostas, salientou-se o recurso à definição da amplitude total e ao estudo de um exemplo. Não existindo estudos prévios acerca desta propriedade, constata-se que os futuros professores dos primeiros anos revelaram um desempenho análogo ao dos alunos do $12 .^{\circ}$ ano do estudo de Boaventura e Fernandes (2004) em relação às propriedades da média e da mediana.

Já no caso da mediana, avaliada através dos itens 1a), 2a) e 2b), observou-se alguma variação na percentagem de respostas corretas de cada um desses itens. No item 1a), à semelhança do que aconteceu no item 1b), mais de quatro em cada cinco estudantes responderam corretamente que a mediana vem aumentada do valor 15 quando se adiciona o valor 15 a todos os dados. Neste item ainda menos estudantes apresentaram justificações para as suas respostas, destacando-se a ideia de que o aumento do valor $15 \mathrm{em}$ todos os dados se repercutiria no aumento do mesmo valor da mediana. Entre os itens 1a) e 1b), verificou-se um 
desempenho um pouco melhor dos estudantes em 1a), o que se poderá dever-se à propriedade da amplitude total ser menos explorada nas aulas.

Nos itens $2 \mathrm{a}$ ) e 2b) os estudantes deviam avaliar o que acontece à mediana quando se acrescenta dois dados em certas condições. Em 2a) acrescentava-se um dado menor e outro maior do que todos os outros, tendo-se constatado que todos os estudantes responderam corretamente. Diferentemente dos dois itens anteriores, quase todos os estudantes justificaram as suas respostas, seja através da análise de exemplos, focando-se no valor central e/ou valores centrais ou focando-se nos valores extremos. Já em 2b), em que se acrescentava um dado com o valor da mediana e outro dado maior do que todos os outros, constatou-se que apenas cerca de um em cada quatro estudantes respondeu corretamente. Tal como no item $2 \mathrm{a}$ ), quase todos os estudantes justificaram as suas respostas, seja através da análise de um exemplo ou afirmando que se mantém/diminui/aumenta para os casos de $n$ ímpar e $n$ par sem darem qualquer explicação.

A grande dificuldade demonstrada pelos estudantes no item 2 b) deve-se ao facto de ser necessário considerar simultaneamente os casos em que o número total de dados é ímpar e par, o que não era requerido no item 2a). Os resultados obtidos são bem expressivos a esse propósito pois quando os estudantes analisaram dois exemplos, um para $n$ ímpar e outro para $n$ par, geralmente, responderam corretamente, enquanto sempre que analisaram apenas um exemplo, quase sempre para $n$ ímpar, geralmente, responderam incorretamente.

Em geral, os resultados obtidos no item 2a) são um pouco superiores aos que foram obtidos em outros estudos que envolviam itens relacionados. No estudo de Boaventura e Fernandes (2004), quase todos os estudantes do $12 .^{\circ}$ ano responderam corretamente ao mesmo item e menos estudantes responderam corretamente a itens envolvendo acrescentar um dado com o valor da mediana ou inferior/superior a todos os dados. Também no caso de acrescentar um dado inferior/superior a todos os outros, Freitas et al. (2018) referem percentagens elevadas de respostas corretas em estudantes do $8 .^{\circ}$ ano, mesmo mais elevadas do que as obtidas pelos alunos do 12. ${ }^{\circ}$ ano do estudo de Boaventura e Fernandes (2004).

Quando era imprescindível estudar os casos de $n$ ímpar e $n$ par, os estudantes, quase sempre, analisaram o caso de $n$ ímpar e responderam incorretamente. Ora, este resultado aprofunda aqueles que foram obtidos em outros itens relativos à mediana do presente estudo e também corroborados em outros estudos, refutando-se, assim, a ideia de que as propriedades da 
mediana são, em geral, fáceis de adquirir. Fernandes, Sousa e Ribeiro (2004) constataram mesmo no seu estudo que a Estatística é considerado um tema fácil tanto para professores como para alunos.

Portanto, de acordo com o presente estudo, assumir que os estudantes não têm grandes dificuldades nas propriedades da mediana, como confirmam alguns itens aqui estudados e a literatura revista, não é uma premissa correta. Antes, recomenda-se que os processos de ensino e aprendizagem enfatizem os dois casos, número total de dados ímpar e par, pois a definição da mediana é diferente em cada um dos casos. Adicionalmente, a análise das várias possibilidades, incluindo não só o número total de dados, mas também o tipo de dados em estudo (têm dados repetidos ou são todos diferentes), como é discutido em Freitas, Cruz e Silva (2017), certamente que também contribuirá para a promoção do desenvolvimento do pensamento estatístico e lógico dos estudantes.

Em conclusão, do presente estudo podemos dizer que as ideias dos futuros professores dos primeiros anos traduzem uma compreensão, aqui entendida como a parte do conhecimento pessoal que coincide com o institucional (Godino \& Batanero, 1994), um tanto limitada das propriedades estatísticas aqui estudadas. Essa limitação revela-se, sobretudo, na ausência de explicações ou de explicações pouco desenvolvidas e/ou incompletas, aspetos que devem ser aprofundados na sua formação inicial.

\section{Referências}

Batanero, C., Godino, J., \& Navas, F. (1997). Some misconceptions about averages in prospective primary teachers. Em E. Pehknonen (Ed.), Proceedings of 21 PME Conference (v. 1, pp. 276). University of Lahti.

Boaventura, M. G., \& Fernandes, J. A. (2004). Dificuldades de alunos do 12. ${ }^{\circ}$ ano nas medidas de tendência central: O contributo dos manuais escolares. Em J. A. Fernandes, M. V. Sousa, \& S. A. Ribeiro (Orgs.), Ensino e aprendizagem de probabilidades e estatística-Actas do I Encontro de Probabilidades e Estatística na Escola (pp. 103-126). Braga: Centro de Investigação em Educação da Universidade do Minho.

Cazorla, I. M. (2003). Média aritmética: um conceito prosaico e complexo. In C. Reading (Ed.). Anais do IX Seminário de Estatística Aplicada (pp. 1-14). Rio de Janeiro: Instituto Interamericano de Estatística.

delMas, R., \& Liu, Y. (2005). Exploring students' conceptions of the standard deviation. Statistics Education Research Journal, 4(1), 55-82. 
Estrada, A., Batanero, C., \& Fortuny, J. M. (2004). Un estudio de evaluación de conocimientos estadísticos en profesores en formación e implicaciones didácticas. Educación Matemática, 16, 89-112.

Fernandes, J. A., \& Barros, P. M. (2005). Dificuldades de futuros professores do $1^{\mathrm{o}}$ e $2^{\mathrm{o}}$ ciclos em estocástica. Actas do $V$ Congresso Ibero-Americano de Educação Matemática (CIBEM). Porto (Portugal): Faculdade de Ciências.

Fernandes, J. A., Batanero, C., \& Gea, M. M. (2019). Escolha e aplicação de métodos estatísticos por futuros professores dos primeiros anos. In J. M. Contreras, M. M. Gea, M. M. LópezMartín, \& E. Molina-Portillo (Eds.), Actas del Tercer Congreso Internacional Virtual de Educación Estadística. Granada: Universidade de Granada.

Fernandes, J. A., Gonçalves, G., \& Barros, P. M. (2021). Uso de tabelas de frequências por futuros professores na realização de trabalhos de projeto. Uniciencia, 35(1), 139-151.

Fernandes, J. A., Sousa, M. V. \& Ribeiro, S. A. (2004). O ensino de estatística no ensino básico e secundário: Um estudo exploratório. Em J. A. Fernandes, M. V. Sousa, \& S. A. Ribeiro (Orgs.), Ensino e aprendizagem de probabilidades e estatística - Actas do I Encontro de Probabilidades e Estatística na Escola (pp. 165-193). Braga: Centro de Investigação em Educação da Universidade do Minho.

Freitas, A., Cruz, J. P., \& Silva, N. (2017). Mediana de dados não agrupados: a questão de ser pelo menos 50\%. Educação e Matemática, 143, 18-21.

Freitas, A., Figueiredo, T. S., Silva, N., \& Miranda, M. C. (2018). Dificuldades na aprendizagem da mediana e quartis por alunos do $8 .^{\circ}$ ano de escolaridade: estudo comparativo fórmula versus gráfico. Indagatio Didactica, 10(2), 109-132.

Godino, J. D. (2002). Un enfoque ontológico y semiótico de la cognición matemática. Recherches en Didactique des Mathématiques, 22(2/3), 237- 284.

Godino, J. D., \& Batanero, C. (1994). Significado institucional y personal de los objetos matemáticos. Recherches en Didactique des Mathématiques, 14(3), 325-355.

Godino, J. D., Batanero, C., \& Font, V. (2007). The onto-semiotic approach to research in mathematics education. ZDM. The International Journal on Mathematics Education, 39(12), 127- 135 .

Godino, J. D., Giacomone, B., Batanero, C., \& Font, V. (2017). Enfoque ontosemiótico de los conocimientos y competencias del profesor de matemáticas. Bolema, 31(57), 90-113.

Hiebert, J., \& Lefevre, P. (1986). Conceptual and procedural knowledge in mathematics: An introductory analysis. Em J. Hiebert (Ed.), Conceptual and procedural knowledge: The case of mathematics (pp. 1-27). Hillsdale, NJ: Lawrence Erlbaum Associates.

Hill, H. C., Ball, D. L., \& Schilling, S. G. (2008). Unpacking pedagogical content knowledge: Conceptualizing and measuring teachers' topic-specific knowledge of students. Journal for Research in Mathematics Education, 39(4), 372- 400.

Jacobbe, T., \& Carvalho, C. (2011). Teachers' understanding of averages. Em C. Batanero, G. Burrill, \& C. Reading (Eds.), Teaching statistics in school mathematics-Challenges for teaching and teacher education (pp. 199-209). Springer, Dordrecht. 
Leon, M. R., \& Zawojewski, J. S. (1991). Use of the arithmetic mean: An investigation of four properties. Issues and preliminary results. Em D. Vere-Jones (Ed.), Proceedings of the Third International Conference on Teaching Statistics (pp. 302-306). Voorburg: International Statistical Institute.

Ministério da Educação (2007). Programa de matemática do ensino básico. Lisboa: Ministério da Educação.

Ministério da Educação e Ciência (2014). Programa de Matemática A - Ensino Secundário. Lisboa: Ministério da Educação e Ciência.

Ministério da Educação e Ciência. (2013). Programa de matemática para o ensino básico. Lisboa, Portugal: Ministério da Educação e Ciência.

Shulman, L. S. (1986). Those who understand: Knowledge growth in teaching. Educational Researcher, 15(2), 3-14.

Strauss, S., \& Bichler, E. (1988). The development of children's concepts of the arithmetic average. Journal for Research in Mathematics Education, 19(1), 64-80.

\section{Autor}

\section{José António Fernandes}

Doutor em Educação pela Universidade do Minho (UM), área de conhecimento de Metodologia do Ensino da Matemática. Professor Associado no Instituto de Educação da Universidade do Minho (UM), Braga, Portugal. Membro do Centro de Investigação em Educação (CIEd) da Universidade do Minho. E-mail: jfernandes@ie.uminho.pt. 